\section{SOI: $1.1 /$ TAS DOI: $10.15863 / \mathrm{TAS}$ \\ International Scientific Journal Theoretical \& Applied Science}

\section{N.M. Kiyalbekov researcher Regional school-internat Daryn nariman_kiyalbekov@mail.ru}

p-ISSN: 2308-4944 (print) e-ISSN: 2409-0085 (online)

Year: $2017 \quad$ Issue: $05 \quad$ Volume: 49

Published: $30.05 .2017 \quad$ http://T-Science.org

SECTION 2. Applied mathematics. Mathematical modeling.
R.A. Beshtaeva researcher

Taraz State University named after M. H. Dulati razila-83@mail.ru

S.S. Kapan

researcher

Taraz State University named after M. H. Dulati

\title{
BASIC EQUATIONS OF TRANSPORT AND DIFFUSION OF POLLUTANTS IN THE ATMOSPHERE
}

Abstract: The structure of the spatial non-stationary numerical model calculating anthropogenic disturbances in the mesoscale meteorological processes is briefly discussed. The results of calculating the microclimate and fog formation variations of cooling reservoirs are presented.

Key words: the air fluctuations, the atmosphere, the model

Language: Russian

Citation: Kiyalbekov NM, Beshtaeva RA, Kapan SS (2017) BASIC EQUATIONS OF TRANSPORT AND DIFFUSION OF POLLUTANTS IN THE ATMOSPHERE. ISJ Theoretical \& Applied Science, 05 (49): 190-192.

Soi: http://s-o-i.org/1.1/TAS-05-49-29 Doi: crossef https://dx.doi.org/10.15863/TAS.2017.05.49.29

\section{ОСНОВНЫЕ УРАВНЕНИЯ ПЕРЕНОСА И ДИФФУЗИИ ПРИМЕСЕЙ В АТМОСФЕРЕ}

Аннотация: Кратко обсуждается структура пространственной нестационарной численной модели расчета антропогенных нарушений в мезомасштабных метеорологических прочессов. Представлень результаты расчета микроклимата и вариаций образование тумана от охлаждения резервуаров.

Ключевые слова: воздух, флуктуачии, атмосфера, модель.

\section{Introduction}

Перенос загрязняющих субстанций в атмосфере осуществляется ветровыми потоками воздуха с учетом их мелкомасштабных флуктуаций. Пусть $\varphi(x, y, z, t)$ - интенсивность аэрозольной субстанции, мигрирующей вместе с потоком воздуха в атмосфере. Решение задачи будем искать в цилиндрической области $G$ с поверхностью $S$, состоящей из боковой поверхности цилиндра $\sum$, нижнего основания $\sum_{0}($ при $z=0)$ и верхного основания $\sum_{H}$ (при $z=H), \quad x, y, z$-декартовы координаты, ось $z$ направлена вертикально вверх. Пусть $u, v, w$ составляющие вектора скорости частиц воздуха. Тогда перенос субстанции вдоль траектории частиц воздуха с сохранением ее интенсивности описывается уравнением переноса:

$$
\frac{d \varphi}{d t}=0, \text { где } \frac{d}{d t}=\frac{\partial}{\partial t}+u \frac{\partial}{\partial x}+v \frac{\partial}{\partial y}+w \frac{\partial}{\partial z} .
$$

\section{Materials and Methods}

Для нижней части атмосферы с хорошей точностью выполняется закон сохранения массы, выраженный уравнением неразрывности:

$$
\operatorname{div} \bar{u}=\frac{\partial u}{\partial x}+\frac{\partial v}{\partial y}+\frac{\partial w}{\partial z}=0 .
$$
виду

С учетом (2) уравнение (1) преобразуется к

$$
\frac{\partial \varphi}{\partial t}+\operatorname{div}(\bar{u} \cdot \varphi)=0
$$

При выводе (2.3) было использовано тождество

$$
u \frac{\partial \varphi}{\partial x}+v \frac{\partial \varphi}{\partial y}+w \frac{\partial \varphi}{\partial z}=\operatorname{div}(\bar{u} \cdot \varphi)-\varphi \operatorname{div} \bar{u} .
$$

Вертикальная составляющая скорости удовлетворяет условию

$$
w=0 \text { при } z=0, z=\mathrm{H} .
$$

Для уравнения (3) необходимо задать начальные данные

$$
\varphi=\varphi_{0} \text { при } \mathrm{t}=0
$$

и условия на границе $\mathrm{S}$ области $\mathrm{G}$ :

$$
\varphi=\varphi_{S} \quad \text { на } \mathrm{S} \quad u_{n}<0,
$$




\begin{tabular}{|c|c|c|c|c|c|c|}
\hline Impact Factor: & $\begin{array}{l}\text { ISRA (India) } \\
\text { ISI (Dubai, UAF } \\
\text { GIF (Australia) } \\
\text { JIF }\end{array}$ & $\begin{array}{l}=1.344 \\
=0.829 \\
=0.564 \\
=1.500\end{array}$ & $\begin{array}{l}\text { SIS (USA) } \\
\text { PИНЦ (Russia) } \\
\text { ESJI (KZ) } \\
\text { SJIF (Morocco) }\end{array}$ & $\begin{array}{l}=0.912 \\
=0.234 \\
=3.860 \\
=\mathbf{2 . 0 3 1}\end{array}$ & $\begin{array}{l}\text { ICV (Poland) } \\
\text { PIF (India) } \\
\text { IBI (India) }\end{array}$ & $\begin{array}{l}=6.630 \\
=1.940 \\
=4.260\end{array}$ \\
\hline
\end{tabular}

где $\varphi_{0}$ и $\varphi_{s}$ - известные функции, $u_{n}$ - проекция вектора $u$ на внешнюю нормаль к поверхности $\mathrm{S}$. Соотношение (7) определяет решение на той части S, где воздушные массы вместе с исследуемой субстанцией вносятся в область G.

Точное решение задачи (3), (5), (7) возможно в том случае, когда известны значения функций $u, v, w$ в пространстве и во времени.

Уравнение (3) может быть обобщено. Так, если в процессе распространения часть субстанции входит в реакцию с внешней средой или распадается, то этот процесс можно интерпретировать как поглощение субстанции. В этом случае уравнение (3) перейдет в следующее:

$$
\frac{\partial \varphi}{\partial t}+\operatorname{div}(\bar{u} \varphi)+\sigma \varphi=0
$$

где $\sigma \geq 0$ - величина, обратно пропорциональная времени. Смысл этой величины будет понятен, если в (8) положить $u=v=w=0$ тогда уравнение (8) имеет решение $\varphi=\varphi_{0} e^{-\sigma t}$. Отсюда видно, что $\sigma$ есть величина, обратная интервалу времени, за который интенсивность субстанции по сравнению с начальной интенсивностью $\varphi_{0}$ уменьшается в $е$ раз.

Если в области определения решения имеются источники рассматриваемой загрязняющей субстанции $\varphi$, описываемые функцией $f(x, y, z, t)$, то уравнение (8) примет вид

$$
\frac{\partial \varphi}{\partial t}+\operatorname{div}(\bar{u} \varphi)+\sigma \varphi=f .
$$

Для достаточно гладких функций задача (9), (5), (7) имеет единственное решение.

Рассмотрим стационарный процесс распространения субстанций. Если коэффициенты уравнений $u, \vartheta, \omega$ и входные данные задачи $\varphi_{S}$ и $f$ не зависят от времени, то стационарная задача, соответствующая (9),(5),(7), формулируется следующим образом:

$$
\begin{gathered}
\operatorname{div}(u \varphi)+\sigma \varphi=f, \\
\varphi=\varphi_{S} \text { на } \sum \text { при } u_{n}<0 .
\end{gathered}
$$

Задача (10), (11) описывает частный процесс переноса субстанций с неизменными во времени входными данными. Однако на бор таких частных решений, соответствующих различным стационарным входным данным задачи $\bar{u}, f, \varphi_{S}$, может использоваться и при описании более сложных физических ситуаций, реализуемых на практике. Предположим, что в различные периоды времени в атмосфере в данном регионе происходят те или иные типы движений воздушных масс, которые за период характерного времени можно считать стационарными. После каждого такого периода происходит перестройка движения воздушных масс и наступает новое стационарное состояние. Поскольку пересторйка циркуляций происходит за период, которой намного короче времени существования данного типа движений, то можно пердположить, что перемена типов движений происходит мгновенно. Пусть этих типов будет $n$. Тогда приходим к системе независимых уравнений

$$
\begin{gathered}
\operatorname{div}\left(\bar{u}_{i} \varphi_{i}\right)+\sigma \varphi_{i}=f \\
\text { и условий } \varphi_{i}=\varphi_{i s} \text { на } \sum_{u_{i n}<0,} \text { при } \\
\quad i=\overline{1, n} .
\end{gathered}
$$

Задача (12), (13), где $\varphi_{i S}$ - значения функции $\varphi_{i}$ на границе $S, u_{i n}$ - проекция вектора скорости ветра $i$-го типа на внешнюю нормаль к границе, соответствует каждому из интервалов времени $t_{i}<t<t_{i+1}$, длина которого $\Delta t_{i}$. Если все задачи (12), (13) решены, то решение задачи о среднем распределении примеси за период $T=\sum_{i=1}^{n} \Delta t_{i}$ находится в виде линейной комбинации

$$
\tilde{\varphi}=\frac{1}{T} \sum_{i=1}^{n} \varphi_{i} \Delta t_{i}
$$

Задачу (12)-(14) можно называть статической моделью.

Решение стационарных задач вида (10), (11) и (12)-(14) имеет много общего с решением задачи о среднем за некоторый период времени $T$ распределении субстанции на основе специальных образом поставленных нестационарных задач. Действительно, рассмотрим задачу

$$
\begin{gathered}
\frac{\partial \varphi}{\partial t}+\operatorname{div}(u \varphi)+\sigma \varphi=f . \\
\varphi=\varphi_{S} \text { на } \sum \quad \text { при } u_{n}<0, \\
\varphi(\bar{r}, T)=\varphi(\bar{r}, 0), \quad \bar{r}=(x, y, z) \in G .
\end{gathered}
$$

Функции $\bar{u}$ и $\varphi_{s}$ не зависят от $t$. Эта задача для достаточно гладких функций имеет единственное решение. Интегрируя (15) в пределах $[0, T]$, получим

$$
\operatorname{div}(u \varphi)+\sigma \varphi=f, \quad \varphi=\frac{1}{T} \int_{0}^{T} \varphi d t
$$

из которого, в силу единственности решения задачи (10), (11), следует, что среднее за период $T$ решение задачи (10), (16) совпадает с решением задачи (10), (11). 


\begin{tabular}{l|lr|ll|ll} 
& ISRA (India) & $=\mathbf{1 . 3 4 4}$ & SIS (USA) & $=\mathbf{0 . 9 1 2}$ & ICV (Poland) & $=\mathbf{6 . 6 3 0}$ \\
Impact Factor: & ISI (Dubai, UAE) $=\mathbf{0 . 8 2 9}$ & PUHIL (Russia) $=\mathbf{0 . 2 3 4}$ & PIF (India) & $=\mathbf{1 . 9 4 0}$ \\
& GIF (Australia) & $\mathbf{0 . 5 6 4}$ & ESJI (KZ) & $=\mathbf{3 . 8 6 0}$ & IBI (India) & $\mathbf{= 4 . 2 6 0}$ \\
& JIF & $=\mathbf{1 . 5 0 0}$ & SJIF (Morocco) & $=\mathbf{2 . 0 3 1}$ & & \\
\hline
\end{tabular}

\section{Conclusion}

Решения задач о среднем за период $T$ распределении субстанций с помощью статической модели и нестационарной задачи с некоторыми предположениями достаточно близки друг к другу.

\section{References:}

1. Marchuk GI, Kondrat'ev KJ, Kozoderov VV, Hvorst'janov VI (1986) Oblaka i klimat. - L., Gidrometeoizdat.

2. Berljand ME (1975) Sovremennye problemy atmosfernoj diffuzii i zagrjaznenija atmosfery. - L., Gidrometeoizdat.

3. Marchuk GI (1982) Matematicheskoe modelirovanie v probleme okruzhajushhej sredy - M.: Nauka.

4. Yamada T (1979) An application of a threedimensional simplifited second - moment closure numerical model to atmospheric effects of large cooling pond. - Atmos. Environ., vol. 13.

5. Hvorst'janov VI (1986) Modelirovanie i shemy zon prosveta pri nazemnom rassejanii pereohlazhdennyh tumanov. - Meteorologija i gidrologija, №3.

6. Vager BG, Nadezhina ED (1979) Pogranichnyj sloj atmosfery $\mathrm{V}$ uslovijah gorizontal'noj neodnorodnosti. - L., Gidrometeoizdat.

7. Orlanski IA (1976) simple boundary condition for unbounded hyperbolic tlows// Y.Comp.Phys.V21.V3

8. Fejgel'son EM (1970) Luchistyj teploobmen i oblaka. - L., Gidrometeoizdat.

9. Krasnokutskaja LD, Sushkevich TA (1977) Analiticheskoe predstavlenie integral'nyh funkcij propuskanija oblakov. - Izv.AN SSSR. Fizika atmosfery i okeana, t.13, № 5. 